\section{Betrachtung von Nachhaltigkeit und Umweltverträglichkeit mithilfe des neuen Syntropie-Index}

\author{
Hochwertige Energieformen wie Solarstrahlung oder elektrischer \\ Strom haben die besondere Fähigkeit, höher geordnete Strukturen \\ und damit die Basis für nachhaltiges Leben im Allgemeinen zu \\ erschaffen. Mit dem neuen Syntropie-Index kann diese Antriebs- \\ kraft auf moderne Systeme wie Gesellschaften oder Unternehmen \\ angewandt und bewertet werden. Von Enno Wagner
}

U m künftige Energie- und Wirtschaftssysteme hinsichtlich ihrer Nachhaltigkeit und Umweltverträglichkeit analysieren und beurteilen zu können, bedarf es einer neuen tiefergehenden Betrachtungsweise. Die Herausforderung besteht in einer allgemeinverständlichen Darstellung von zwei gegensätzlich wirkenden physikalischen Antriebskräften: Der Entropie und der Syntropie. Während die Entropie eine möglichst gleichmäßige, großflächige und energiearme Verteilung anstrebt, bewirkt die Syntropie die Schaffung von energiereichen Inseln, angefüllt mit Vielfalt und Artenreichtum. Die Wüste steht gegenüber dem Regenwald - der Tod gegenüber dem Leben.

Nach dem zweiten Hauptsatz der Thermodynamik kann die Entropie (anschaulich: „die Unordnung“) in einem geschlossenen System mit der Zeit nur zunehmen, niemals freiwillig abnehmen. Denken wir uns hierzu beispielsweise einen geschlossenen Raum, in dem sich eine Anzahl Kinder frei bewegen kann. Ist nun in einer Ecke des Raumes eine feinsäuberliche Anordnung von farbigen Bauklötzen aufgebaut, so ist dies über die Zeit hinweg gesehen ein unwahrscheinlicher Zustand. Viel wahrscheinlicher ist es, dass die Bauklötze nach einer gewissen Zeit bunt gemischt und quer über die Bodenfläche des Raumes verstreut herumliegen. Beobachtet man die Kinder über diese Zeit, so wird man feststellen, dass sich eine Art dest- ruktives "Spiel“ entwickeln wird, durch das die Bauklötze bestmöglich der maximalen Verstreuung zustreben.

\section{Von Destruktiv bis Kreativ}

Im zweiten Teil des Gedankenexperimentes öffnet man nun den Raum und versorgt die Kinder mit hinreichend hochwertiger Syntropie in Form von Essen und Trinken, Musik, Papier, Klebstoff, Farben und entsorgt gleichzeitig die niederwertige Unordnung, indem man eine Toilette bereitstellt und regelmäßig den Müll entfernt. Mit großer Wahrscheinlichkeit wird nun das kreative Potenzial freigesetzt und schöpferische Kräfte angeregt. Als Resultat wird man Unmengen fröhlicher Bilder und eine Vielzahl erfinderisch gebastelter Dinge und auch neu geschaffene bunte Bauklotz-Türme bewundern können.

So ähnlich verhält es sich auch mit dem System Erde. Die riesigen unterirdischen Blasen, gefüllt mit hoch konzentrierten Energiestoffen wie Erdöl, stellen zeitgeschichtlich gesehen einen sehr unwahrscheinlichen Zustand dar. Viel wahrscheinlicher ist es, dass das Erdöl mit der Zeit möglichst gleichmäßig über den Globus verteilt, vollständig verbrannt und die Verbrennungsrückstände in der Atmosphäre zerstreut werden. Doch hat die Natur wenig wirksame Mechanismen für diese Maximierung der Entropie. Hier und da sprudelt etwas Erdöl hervor, um nebenan wieder zu versickern.
Eine offene Ölquelle, die sich rein zufällig durch einen Blitz entzündet, ist ein eher außergewöhnliches Ereignis. Doch der Mensch mit seiner Technologie hilft hier auf einmal dem Bedürfnis der Natur! Er erfindet Methoden zur globalen Verteilung und Verbrennung des Rohstoffes. Mit seinem „Spiel“ hilft er dem thermodynamischen Verlangen zur optimierten Maximierung von Entropie. Und je wirkungsvoller der Verteilmechanismus läuft, umso kräftiger sprudeln offensichtlich die Quellen und umso schneller schnurrt seine Maschinerie.

Es ist wie ein See auf der Spitze eines Sandberges: Einmal angeritzt gräbt sich das Wasser immer tiefere Furchen und Bäche und läuft immer unaufhaltsamer bergab. Es besteht nun der Verdacht, dass die Verarbeitungs- und Verteilsysteme der modernen Petrochemie vor allem diesem natürlichen Drang und enormen Druck zur Maximierung der Entropie folgen und dass die dabei entspringenden Produkte und Profite nur vordergründig nützlich für den Menschen sind. Es sind entropische Systeme. Die produzierte Entropie zeigt ihr Gesicht in Form der „Nebeneffekte“. So breiten sich Wüsten und Steppen von Monokulturen aus und die Schadstoffe der Verbrennungsprodukte drohen unsere Biosphäre zu vergiften und den Planeten zu überhitzen.

\section{Unordnung im System Erde}

Zum Glück ist die Erde jedoch kein geschlossenes System. Auf der einen Seite erhält sie von der Sonne einen kontinuierlichen Zustrom an hochwertiger UV-Strahlung. Diese Solarenergie enthält eine hohe Exergie [1]. Auf der anderen Seite strahlt die Erde niederwertige Infrarotstrahlung in den Weltraum ab, die wiederum eine hohe Entropie besitzt. Allgemeinverständlich kann man also zusammenfassen: Die Erde erhält niedrige Unordnung von der Sonne und entsorgt hohe Unordnung in den Weltraum, es verbleibt eine „negative Unordnung" auf der Erde. Diese negative Unordnung erzeugt quasi einen Antrieb zu höherer Ordnung, zu der ganz allge- 


\begin{tabular}{|c|c|c|c|c|c|c|c|}
\hline Unternehmen & Branche & Patente & $\begin{array}{l}\text { Energie gesamt } \\
\text { GWh }\end{array}$ & $\begin{array}{l}\text { elektrische Energie } \\
\text { GWh }\end{array}$ & $\begin{array}{l}\text { Wärmeenergie } \\
\text { GWh }\end{array}$ & $\begin{array}{l}\text { Exergie } \\
\text { GWh }\end{array}$ & $\begin{array}{l}\text { Syntropie-Index } \\
\text { Patente/GWh }\end{array}$ \\
\hline Beispiel 1 & Chemie & 154 & 18.073 & 1.692 & 16.381 & 8.244 & 0,02 \\
\hline Beispiel 2 & Automobil & 1.854 & 9.516 & 4.545 & 4.971 & 6.533 & 0,28 \\
\hline Beispiel 3 & Zulieferer & 708 & 2.646 & 1.575 & 1.071 & 2.003 & 0,35 \\
\hline Beispiel 4 & Mechatronik & 4.144 & 6.200 & 3.720 & 2.480 & 4.712 & 0,88 \\
\hline Beispiel 5 & Mikroelektronik & 1.784 & 1.188 & 1.117 & 71 & 1.145 & 1,60 \\
\hline Quellen: & $\begin{array}{l}\text { Jahresbericht } 2013 . \\
\text { Jahresberichte der B } \\
\text { Die präsentierten Zc }\end{array}$ & $\begin{array}{l}\text { hes Pate } \\
\text { interneh } \\
\text { eruhent }\end{array}$ & $\begin{array}{l}\text { rkenamt, Münche } \\
\text { uere Quellenangal } \\
\text { feigenen Berechnu }\end{array}$ & $\begin{array}{l}\text { 4). www.dpma.de. } \\
\text { fAnfrage erhältlich). } \\
\text { durch den Autor. }\end{array}$ & & & \\
\hline
\end{tabular}

Abbildung 1: Syntropie-Index für verschiedene Unternehmen aus dem produzierenden Gewerbe.

Quelle: Autor, mit Daten des DPMA sowie der Unternehmensberichte (DPMA 2014)

mein das Leben zählt. Man kann auch sagen: Es wird hochwertige Energie zugeführt und niederwertige Energie abgeführt. Also verbleibt die „Hochwertigkeit“ auf der Erde. Diese antreibende Kraft der negativen Entropie und der gewonnenen Hochwertigkeit wird als Negentropie oder auch Syntropie bezeichnet [2]. Syntropie ist demnach schöpferisch. Es ist kreative Energie, die höher geordnete Strukturen, neuartige Verbindungen und damit Leben schafft, das ausgezeichnet ist durch Vielfältigkeit, Anpassungsfähigkeit und Artenreichtum (Dürr 2011, Bergmann 1977). Ein syntropisches System ist allgemein ein System mit einem kontinuierlichen Zustrom an hochwertiger Syntropie und einem kontinuierlichen Abstrom an niederwertiger Entropie. Im Inneren des syntropischen Systems bilden sich komplexe und neuartige Strukturen aus. Auch Gemeinschaften, Unternehmen, Städte und Länder können demnach syntropische Systeme sein, wenn die Energie- und Stoffströme über die Systemgrenzen bestimmt werden können.

In modernen und auch künftigen Gesellschaften werden immer Mischformen aus entropischen und syntropischen Systemen auftreten, da auch fossile Energien hochwertige Anteile haben und Syntropie enthalten. Es geht folglich um eine Gewichtung und zunächst grobe Einordnung der Systeme. Während entropische Systeme vor allem auf einen maximalen Durchsatz ausgerichtet sind, steht bei syntropischen Systemen die Schaffung von Neuem und die Vielfältigkeit im Vordergrund.

Ein Vorschlag zur Charakterisierung ist nun der syntropische Index oder auch
Syntropie-Index (engl.: Syntropic Number, $\mathrm{SN}$ ). Er ist eine charakteristische Kennzahl und einfacher Weise gekennzeichnet durch das Verhältnis von Innovationen pro Energieumsatz:

$$
S N=\frac{\text { Number of Inventions }}{\text { Quantity of Exergy }}=\frac{N_{\text {inv }}}{E_{e x}}\left[\frac{1}{J}\right]
$$

In Abbildung 1 sind beispielhaft die syntropischen Indizes von fünf modernen Unternehmen dargestellt. Die Ergebnisse basieren auf einer durch den Autor durchgeführten und bislang noch nicht veröffentlichten Studie. Als Berechnungsgrundlage wurden die Patentstatistik des DPMA sowie die in den Unternehmensberichten veröffentlichten Zahlen für Innovationen (angemeldete Patente) und für den Gesamtenergieeinsatz pro Jahr verwendet. Es wurden beispielhaft fünf Unternehmen ausgewählt, die repräsentativ für den entsprechenden Bereich sind.

\section{Bewertung von Unternehmen}

Es fällt auf, dass Unternehmen 1 aus dem Bereich Chemie einen vergleichsweise niedrigen Syntropie-Index aufweist. Bei den Prozessen der Spezialchemie werden sehr große Mengen fossiler Brennstoffe eingesetzt, um Tonnagen von beispielsweise Füllstoffen für Lebensmittel herzustellen. Der nachhaltige Nutzen für künftige Generationen scheint hierbei gering zu sein, die Belastung der Umwelt durch Schadstoffe dagegen hoch. Bei der Produktion riesiger Mengen von Pestiziden hingegen entstehen sogar zusätzliche noch massivere und langfristigere Schäden für nach- folgende Generationen durch Artensterben, Monokulturen und daraus folgende Versteppung und Verarmung der Bevölkerung. Hier scheint die Entropieproduktion in denkbar hohem Ausmaß den Prozess zu dominieren. Die Anpassungsfähigkeit dieser Unternehmen auf sich wandelnde Energieformen scheint fragwürdig.

Die aufgeführten Unternehmen 2 und 3 aus dem Bereich Automobilbau und Zulieferindustrie stellen aktuell den Stand der Technik unserer Gesellschaft dar. Hier werden noch große Mengen fossiler Brennstoffe eingesetzt, da die gesamte automobile Infrastruktur auf die Verteilung des energiereichen und leichten Erdöls ausgerichtet ist. Es ist folglich ein entropiegetriebenes System. Auf der anderen Seite wird positiverweise bereits ein nennenswerter Teil der Energie in die Erforschung zukunftsfähiger Technologien investiert. Der Umbau der Automobilindustrie auf elektrische Antriebe wird in jedem Fall die größte Herausforderung seit Beginn ihres Bestehens.

Die Unternehmen 4 und 5 aus den Bereichen Mechatronik/Elektronik zeichnen sich durch einen hohen syntropischen Index aus und haben bemerkenswerterweise auch einen hohen Anteil an Strom in ihrem Energiemix. Sie können am ehesten auf Solarstrom umstellen und haben es bereits teilweise getan. Zudem scheint hier die Innovation gegenüber dem reinen Energieumsatz zu überwiegen. Diese Unternehmen generieren einen hohen Mehrwert für nachfolgende Generationen, da sie in ihrer Produktpalette sehr breit aufgestellt sind und viele Neuerungen für sich wandelnde Energieformen bereitstellen. 
Doch auf der anderen Seite sollten auch sehr hohe Syntropie-Indizes kritisch betrachtet werden, die in naher $\mathrm{Zu}$ kunft im Bereich Digitalisierung auftreten könnten. So investieren aktuell einige namhafte amerikanische Großunternehmen der Informationstechnologie massiv in die Bereiche „Internet der Dinge“, Cloud-Anwendungen und künstliche Intelligenz. Die Zahl der Patente hierzu ist bei einem Unternehmen im Jahr 2017 zum ersten Mal auf über 9.000 angestiegen. Jene Großunternehmen erschaffen gerade ein weltumspannendes Netzwerk aus Mega-Rechenzentren, die über Glasfaserleitungen in Lichtgeschwindigkeit miteinander kommunizieren und mit Solarstrom versorgt werden. Diese "Cloud“ ist ein thermodynamisches System, das kontinuierlich mit hochwertigster Energie gefüttert wird und über die Zeit mit nennenswerter Wahrscheinlichkeit neuartige Strukturen höherer Ordnung und Intelligenz hervorbringen wird, die wir dann vielleicht nicht mehr so leicht beherrschen können.

\section{Kennzahl der Nachhaltigkeit}

Zusammenfassend gesagt, ist der syntropische Index folglich eine Kennzahl, die in einfacher Weise zur Charakterisierung der Nachhaltigkeit und Umweltverträglichkeit von Systemen mit klar definierten Systemgrenzen angewendet werden kann. Er gibt Aufschluss darüber, ob ein System eher fossil ausgerichtet und von hohem Massendurchsatz geprägt ist (niedriger Index) oder ob bei geringer Abhängigkeit von der Energieform die Innovation im Vordergrund steht (hoher Index). Systeme mit vergleichsweise hohem Index sind daher grundsätzlich nachhaltiger ausgerichtet und eher in der Lage sich umweltverträglich anzupassen.

Zur weiteren Validierung sollte der Syntropie-Index im Rahmen einer Studie auf eine größere Anzahl technisch/ wirtschaftlicher Systeme angewandt und die Ergebnisse analysiert und strukturiert werden. Der Syntropie-Index sollte zudem keine absolute Größe sein, da moderne Systeme durch eine Vielzahl weitere Faktoren gekennzeichnet sind, die im Zweifelsfall mit heranzuziehen sind. Absolute Grenzwerte sollten immer mit Vorsicht betrachtet werden. Der syntropische Index kann vielmehr hilfreiche Vergleichs- oder Richtwerte liefern, mit denen eine allgemeine Tendenz oder eine zeitliche Entwicklung angegeben werden kann.

\section{Anmerkugnen}

[1] Exergie ist die Arbeitsfähigkeit einer Energieform. Elektrischer Strom oder heiße Gase haben eine hohe Arbeitsfähigkeit zum Antrieb von Maschinen. Umgebungswärme ist niederwertig, mit ihr kann man keine Maschine mehr antreiben - sie hat eine hohe Entropie.

[2] Chemie-Lexikon: „Der Begriff Syntropie wurde 1944 von dem italienischen Mathematiker Luigi Fantappié in die wissenschaftliche Diskussion eingeführt und bezeichnet dort die Fähigkeit lebender Systeme, sich auf einen in der Zukunft liegenden Zustand besserer Organisation auszurichten. Im Gegensatz zum Begriff der Entropie, mit dem man davon ausgeht, dass Systeme zu einem Ausgleich der Energieniveaus tendieren, ist Syntropie charakterisiert durch die Konzentration von Energie, durch Ordnung und die Herstellung von Differenzen, sowie die Fähigkeit eines Systems gegen den entropischen Tod zu arbeiten. " (Lumitos 2018)

\section{Literatur}

Bergmann, J. (1977): Albert Szent Gyorgyi's theory of Syntropy and Creationizm. Acts \& facts 612

DPMA (2014): Jahresbericht 2013. German Patent and Trade Mark Office, München.

Dürr, H. D. (2011): Das Lebendige lebendiger werden lassen. Oekom Verlag, München.

Lumitos (2018): Online-Lexikon CHEMIE.DE, Lumitos $\mathrm{GmbH}$, Berlin. Im Internet unter www.chemie.de/lexikon/Syntropie

\section{AUTOR + KONTAKT}

Dr. Enno Wagner arbeitet seit seiner Promotion im Fach Thermodynamik in verantwortungsvollen Positionen in der Industrie, unter anderem in den Bereichen Chemie, Erneuerbare Energien, Umweltsimulation und Digitalisierung.

Telefon: +4961316341696, E-Mail: enno.wagner@gmx.de

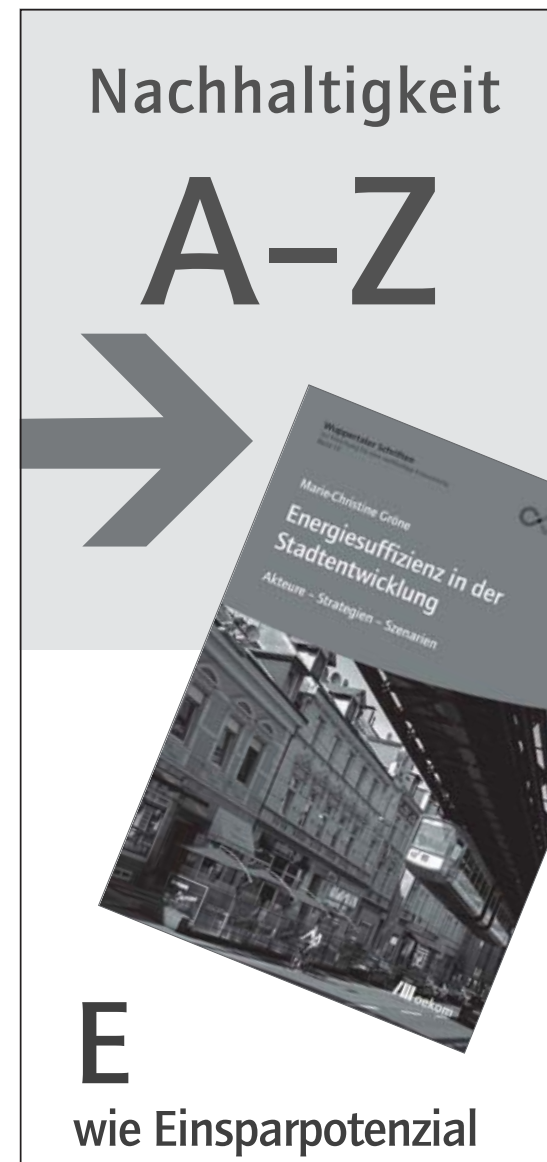

Unsere Kohlendioxidemissionen müssen verringert werden - massiv und schnell. Derzeit geht es da v. a. um mehr Energieeffizienz und mehr erneuerbare Energien. Diese Studie untersucht eine bislang zu wenig beachtete dritte Strategie: Energiesuffizienz. Am Beispiel eines Wuppertaler Stadtteils werden dafür lokale Akteure, geeignete Strategien und das erreichbare Einsparpotenzial an Energie und Kohlendioxid analysiert.

M.-Ch. Gröne

Energiesuffizienz in der Stadtentwicklung Akteure - Strategien - Szenarien 372 Seiten, broschiert, 40,- Euro, ISBN 978-3-96238-069-4

Erhältlich im Buchhandel oder versandkostenfrei innerhalb Deutschlands bestellbar unter www.oekom.de. Auch als E-Book erhältlich.

\section{/II oekom}

Die guten Seiten der Zukunft 